\title{
On the entanglement entropy for a XY spin chain
}

\author{
Ingo Peschel \\ Fachbereich Physik, Freie Universität Berlin, \\ Arnimallee 14, D-14195 Berlin, Germany

\begin{abstract}
The entanglement entropy for the ground state of a XY spin chain is related to the
\end{abstract} \\ corner transfer matrices of the triangular Ising lattice and expressed in closed form.
}

In the study of the properties of quantum states the entanglement entropy plays an important rôle. It is calculated from the reduced density matrix for part of the system and measures how many states are connected across the interface between both parts. For critical one-dimensional systems, it diverges logarithmically with the size of the subsystem, and the prefactor of the logarithm involves the conformal anomaly c. [1,2]. This has been checked numerically for a number of solvable spin chains recently $[3,4]$. Analytical calculations exist for for the (critical) XX chain (equivalent to free fermions hopping on a lattice) [5] and for the (non-critical) XY chain in a field [6], as well as for the ferromagnetic Heisenberg chain [7]. In the first two cases, the limit of a large subsystem was treated by using asymptotic properties of the Toeplitz matrices which determine the density matrix eigenvalues. For the XY chain, the entropy was thereby obtained as an integral over theta functions. In the present note we want to point out that the XY chain can also be treated by connecting it to a triangular Ising model and its corner transfer matrices. Moreover, a much simpler formula for the entropy can be given (at least for part of the parameter space) which involves only elliptic integrals.

Our aim is to study the ground state $\mid 0>$ of the XY Hamiltonian

$$
H=-\sum_{n}\left[(1+\gamma) \sigma_{n}^{x} \sigma_{n+1}^{x}+(1-\gamma) \sigma_{n}^{y} \sigma_{n+1}^{y}+2 h \sigma_{n}^{z}\right]
$$

where the $\sigma_{n}^{\alpha}$ are Pauli matrices and $0 \leq \gamma \leq 1, h \geq 0$. This operator can be obtained from

$$
\hat{H}=-\sum_{n}\left[(1+\gamma) \tau_{n}^{x}-(1-\gamma) \tau_{n-1}^{z} \tau_{n}^{x} \tau_{n+1}^{z}+2 h \tau_{n}^{z} \tau_{n+1}^{z}\right]
$$

where the $\tau_{n}^{\alpha}$ are also Pauli matrices by the dual transformation $\tau_{n}^{x}=\sigma_{n-1}^{x} \sigma_{n}^{x}, \quad \tau_{n}^{z} \tau_{n+1}^{z}=\sigma_{n}^{z}$. The ground state of $\hat{H}$ will be denoted by $\mid \hat{0}>$. It is important to make this distinction because the dual transformation affects the entanglement properties. The bond variable $\sigma^{z}$ only registers the relative orientation of the neighbouring $\tau^{z}$-spins.

Now it is known that $\hat{H}$ commutes with the symmetrized transfer matrix $T$ of a triangular Ising model shown in Fig. 1 [8-10]. Moreover, $\mid \hat{0}>$ corresponds to the maximal eigenvalue of $T$. The relation of the three coupling constants $K_{i}=\beta J_{i}$ of the triangular lattice and the chain parameters is

$$
\gamma=1 / C_{3}, \quad h=\left(S_{1} S_{2} C_{3}+C_{1} C_{2} S_{3}\right) / C_{3}
$$

where $S_{i}=\sinh 2 K_{i}$ and $C_{i}=\cosh 2 K_{i}$. The ordered region of the Ising system, $T<T_{c}$, corresponds to $h>1$, the disordered one to $h<1$. For $K_{3}=0$, one is dealing with a square lattice. Then $\gamma=1$ and $\hat{H}$ becomes the Hamiltonian of an Ising chain in a transverse field. This system was studied in [11] by noting that the reduced density matrix $\hat{\rho}$ for a half-chain is the partition function of a long Ising strip 
with a perpendicular cut. If the chain length resp. the strip width are much larger than the correlation length, this partition function can be expressed in terms of infinite corner transfer matrices (CTM's).

The same can be done for the triangular lattice. In this case one multiplies alternatingly CTM's with a central spin $\left(A_{i}\right)$ and without one $\left(H_{i}\right)$ as shown in Fig. 2. These quantities were studied in $[12,13]$. The result is that $\hat{\rho}$ has the diagonal form

$$
\hat{\rho}=\mathcal{K} \exp \left(-\sum_{j \geq 0} \hat{\varepsilon}_{j} c_{j}^{\dagger} c_{j}\right)
$$

with fermionic operators $c_{j}^{\dagger}, c_{j}$ and single particle eigenvalues

$$
\hat{\varepsilon}_{j}=\left\{\begin{array}{c}
(2 j+1) \varepsilon, \quad T>T_{c} \\
2 j \varepsilon, \quad T<T_{c}
\end{array}\right.
$$

The factor $\varepsilon$ which sets the scale is $\varepsilon=\pi I\left(k^{\prime}\right) / I(k)$ where $I(k)$ denotes the complete elliptic integral of the first kind and $k^{\prime}=\sqrt{1-k^{2}}$. The modulus $k$, finally, is the Onsager parameter for the triangular lattice and given by eqn. (6.4.16) in Ref. [14]. In terms of the chain parameters one finds, using (3) and choosing $K_{1}=K_{2}$, that

$$
k= \begin{cases}\sqrt{h^{2}+\gamma^{2}-1} / \gamma, & h<1 \\ \gamma / \sqrt{h^{2}+\gamma^{2}-1}, & h>1\end{cases}
$$

To obtain the reduced density matrix $\rho$ for the XY chain, one notes that the dual transformation interchanges order and disorder. Therefore one has to interchange the expressions for the eigenvalues in (5) to obtain

$$
\varepsilon_{j}=\left\{\begin{array}{c}
2 j \varepsilon, \quad h<1 \\
(2 j+1) \varepsilon, \quad h>1
\end{array}\right.
$$

In the special case of the transverse Ising chain $(\gamma=1)$ this spectrum coincides with the one obtained directly from (2) and (4). The eigenvalue $\varepsilon_{0}=0$ describes the (asymptotic) degeneracy of the density matrix eigenfunctions for $h<1$ connected with the spin inversion $\sigma^{x} \rightarrow-\sigma^{x}$.

This is valid for purely ferromagnetic interactions as well as for antiferromagnetic competing interactions in the Ising model. If we confine ourselves to the first case, $h$ and $\gamma$ satisfy $h^{2}+\gamma^{2}>1$ and $k$ is real, $0 \leq k \leq 1$. Thus one never crosses the "disorder line" $h^{2}+\gamma^{2}=1$ in the $\gamma-h$-plane. In the terminology of [6] one is in the regions (1a) and (2). In these regions we have completely determined the reduced density matrix. Comparing with Ref. [6] one sees that the eigenvalues $\varepsilon_{j}$ correspond to the quantities $\lambda_{j}$ there. Moreover, $\varepsilon$ is precisely the quantity $\pi \tau_{0}$, as can be seen by evaluating the integrals for $\tau_{0}$. The two approaches therefore give the same density-matrix spectrum, as they should, but the way via corner transfer matrices provides more physical insight.

Given the thermal form of the reduced density matrix, the entanglement entropy $S=-\operatorname{tr}(\rho \ln \rho)$ can immediately be written down

$$
S=\sum_{j} \ln \left[1+\exp \left(-\varepsilon_{j}\right)\right]+\sum_{j} \frac{\varepsilon_{j}}{\exp \left(\varepsilon_{j}\right)+1}=\ln Z+U
$$


This expression has been evaluated numerically and analysed near criticality (where $\varepsilon$ goes to zero) in [2]. However, one can also obtain a closed form by introducing the elliptic nome

$$
q=\exp (-\varepsilon)=\exp \left(-\pi I\left(k^{\prime}\right) / I(k)\right)
$$

and invoking elliptic function identities. In the case $h>1$, one finds the following formula for $Z$ from equ.(16.37.3) in [15]

$$
\prod_{j=0}^{\infty}\left(1+q^{2 j+1}\right)=\left(\frac{16 q}{k^{2} k^{\prime} 2}\right)^{1 / 24}
$$

while for the sum in the "internal energy" $U$, equation (16.23.11) in [15] leads to

$$
\sum_{j=0}^{\infty}(2 j+1) \frac{q^{2 j+1}}{1+q^{2 j+1}}=\frac{1}{24}\left[1-\left(1-2 k^{2}\right)(2 I(k) / \pi)^{2}\right]
$$

Similar formulae hold for the case $h<1$. In this way one arrives at the results

$$
\begin{gathered}
S=\frac{1}{12}\left[\ln \left(\frac{k^{2}}{16 k^{\prime}}\right)+\left(1-\frac{k^{2}}{2}\right) \frac{4 I(k) I\left(k^{\prime}\right)}{\pi}\right]+\ln 2, \quad h<1 \\
S=\frac{1}{24}\left[\ln \left(\frac{16}{\left(k^{2} k^{\prime 2}\right)}+\left(k^{2}-k^{\prime 2}\right) \frac{4 I(k) I\left(k^{\prime}\right)}{\pi}\right], \quad h>1\right.
\end{gathered}
$$

where the $\ln 2$ in $(12)$ arises from the eigenvalue $\varepsilon_{0}=0$. Near criticality $(h \rightarrow 1, k \rightarrow 1)$ these formulae give the logarithmic divergence

$$
S \approx \frac{1}{12} \ln \left(\frac{1}{1-k}\right)
$$

found in [2] where the prefactor corresponds to the value $c=1 / 2$ of the central charge. For $k \rightarrow 0$, the brackets vanish in both cases. If $h \leq 1$, this happens as one approaches the disorder line. Then $\mid 0>$ becomes the superposition of two product states [10] and $S$ goes to $\ln 2$. The corresponding divergence of the $\varepsilon_{j}$ for $j \neq 0$, which leads to a collapse of the spectrum of $\rho$, has been seen in numerical calculations for small systems [16]. A particular case is $\gamma=1$, where one approaches for $h \rightarrow 0$ a superposition of the two states with all spins in the positive resp. negative $x$-direction. For $h>1$, the parameter $k$ vanishes as $h \rightarrow \infty$. Then $\mid 0>$ becomes the simple product state $\mid+++\ldots . .>$ and the entropy vanishes.

All these results refer to a chain with open ends which is divided into two parts. These two parts have one point of contact. If one cuts a segment out of a long chain or a ring, which is the situation studied in [6], one creates two such interfaces. Since the density matrix eigenfunctions for the low $\varepsilon_{j}$ are localized near an interface, one obtains a double degeneracy of the $\varepsilon_{j}$ with $j \neq 0$ if the segment is longer than the correlation length. This degeneracy was also noted in [6] and gives a factor of two for the brackets in (12) and (13), while the logarithm is unchanged.

We have not discussed the region $\gamma^{2}+h^{2}<1$ inside the disorder line (the region (1b) in [6]). The CTM's for this case have not been studied so far. However, on the line $h=0$ one can relate the Hamiltonian of the XY chain to the transfer matrix of a doubled square lattice, i. e. an eight-vertex model at the decoupling point $[17,14]$. For this case the CTM's are known again [14]. One then obtains $\varepsilon_{j}=j \varepsilon$ and the value for $k$ is $k=(1-\gamma) /(1+\gamma)$. This corresponds again to the result for $\pi \tau_{0}$ in [6]. Since now even 
and odd multiples of $\varepsilon$ enter, the entropy for a chain cut in half becomes the sum of the two expressions (12) and (13). The divergence of $S$ in the isotropic limit $\gamma \rightarrow 0, k \rightarrow 1$ is therefore also stronger than (14) by a factor of two, in accordance with the value $c=1$ for this critical point.

Acknowledgement. The author thanks K. D. Schotte for providing the two figures and B. Davies for discussions.

[1] Ch. Holzhey, F. Larsen and F. Wilczek, Nucl. Phys. B 424, 443 (1994)

[2] P. Calabrese and J. Cardy, J. of Statistical Mechanics, P06002 (2004)

[3] G. Vidal, J. I. Latorre, E. Rico and A. Kitaev, Phys. Rev. Lett. 90, 227902 (2003)

[4] J. I. Latorre, E. Rico and G. Vidal, Quant. Inf. and Comp. 4, 48 (2004), preprint: quant-ph/0304098

[5] B.-Q. Jin and V. E. Korepin, J. Stat. Phys. 116, 79 (2004), preprint: quant-ph/0304108

[6] A. R. Its, B.-Q. Jin and V. E. Korepin, preprint quant-phys/0409027

[7] V. Popkov and M. Salerno, preprint quant-phys/0404026

[8] M. J. Stephen and L. Mittag, J. Math. Phys. 13, 1944 (1972)

[9] S. Krinsky, Phys. Lett. 39 A, 169 (1972)

[10] I. Peschel and V. J. Emery, Z. Physik B 43241 (1981)

[11] I. Peschel, M. Kaulke and Ö. Legeza, Ann. Physik (Leipzig) 8, 153 (1999)

[12] S. K. Tsang, J. Stat. Phys. 17, 137 (1977)

[13] R. J. Baxter, J. Stat. Phys. 70, 535 (1993)

[14] R. J. Baxter, Exactly Solved Models in Statistical Mechanics, Academic Press, London (1982)

[15] M. Abramowitz and I. Stegun, (eds.) Handbook of Mathematical Functions, Dover, New York (1965)

[16] M.-C. Chung and I. Peschel, Phys. Rev. B 64, 064412 (2001)

[17] B. Sutherland, J. Math. Phys. 11, 3183 (1970) 


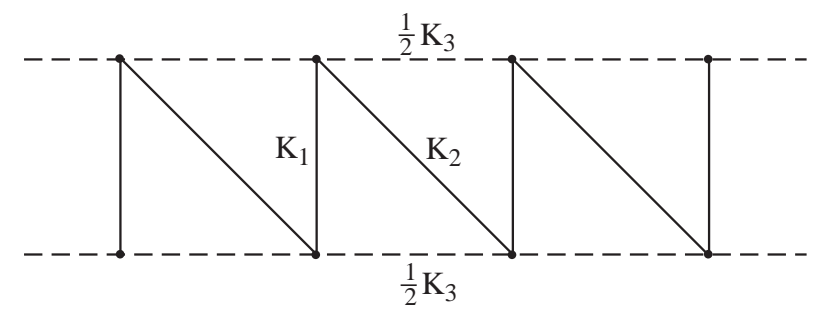

FIG. 1. Portion of a triangular lattice forming the row transfer matrix $\mathrm{T}$

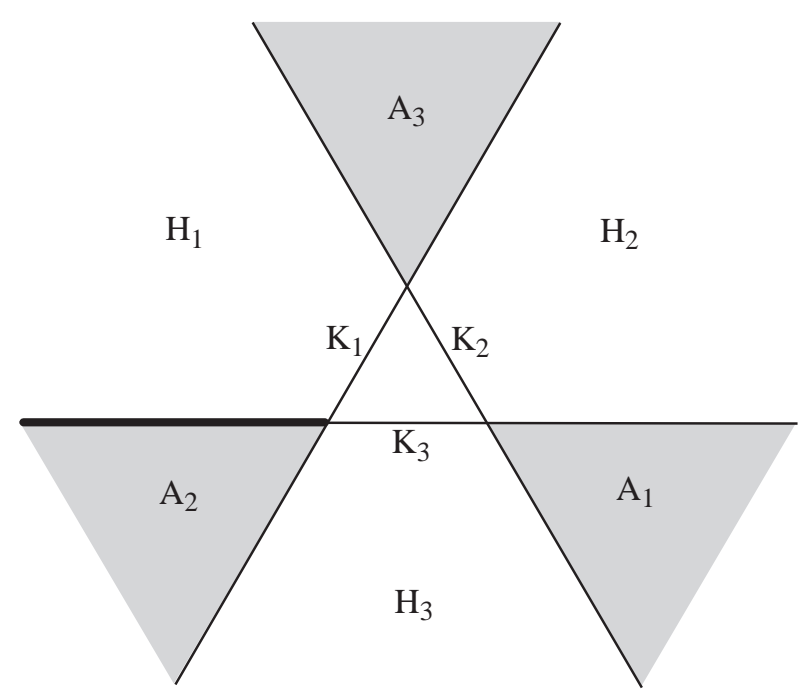

FIG. 2. Construction of the reduced density matrix $\hat{\rho}$ from six triangular corner transfer matrices. Along the heavy line the lattice is cut open. The notation follows Ref. [13]. 